\title{
$\mathrm{FePS}_{3}$ 纳米片制备及其体外光热一光动力学联合治疗性能研究
}

\author{
杨 励 ${ }^{1,3}$, 朱 敏 ${ }^{1}$, 陈 雨 ${ }^{2}$, 朱钰方 ${ }^{1,3}$
}

(1. 上海理工大学 材料科学与工程学院, 上海 200093; 2. 上海大学 生命科学学院, 上海 $200444 ; 3$. 中国科学院 上海硅酸盐研究所, 上海 200050)

摘 要: 光学治疗作为一种肿瘤治疗策略具有微创、毒副作用小、治疗效率高等优势而得到广泛研究, 但单一光学 治疗并不能完全消除肿瘤。新兴的二维纳米材料在光学治疗领域的优势引起了广泛关注。本研究探索了金属磷三 硫族元素化合物 $\mathrm{FePS}_{3}$ 纳米片的制备及其多功能光学治疗性能。采用高温固相法合成 $\mathrm{FePS}_{3}$ 块体并通过超声协助的 液相剥离法得到 $\mathrm{FePS}_{3}$ 纳米片, 该纳米片的平均水合粒径小于 $200 \mathrm{~nm}$ (平均 $153 \mathrm{~nm}$ ), 对 $1064 \mathrm{~nm}$ 激光的光热转换 效率为 $19.7 \%$, 且能在 $660 \mathrm{~nm}$ 激光辐照下产生活性氧。细胞实验结果表明, $\mathrm{FePS}_{3}$ 纳米片具有良好的光热治疗和光 动力学治疗效果。因此, $\mathrm{FePS}_{3}$ 纳米片可同时作为光热剂和光敏剂获得光热-光动力学联合治疗肿瘤功能, 肿瘤治疗 应用潜力较大。

关 键 词: 二维纳米材料; $\mathrm{FePS}_{3}$ 纳米片; 光学治疗; 联合治疗

中图分类号: TQ174 文献标志码: A

\section{FePS $_{3}$ Nanosheets: Preparation and Potential in Photothermal-photodynamic Therapy}

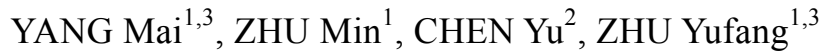

(1. School of Materials Science and Engineering, University of Shanghai for Science and Technology, Shanghai 200093, China; 2. School of Life Sciences, Shanghai University, Shanghai 200444, China; 3. Shanghai Institute of Ceramics, Chinese Academy of Sciences, Shanghai 200050, China)

\begin{abstract}
Phototherapy, a tumor therapeutic modality, has been widely studied due to its advantages of minimal invasiveness, low side effect, and high therapeutic efficacy. However, single phototherapy cannot achieve complete tumor elimination. Recently, the emerging two-dimensional nanomaterials have attracted great attention in the field of phototherapy. Here, $\mathrm{FePS}_{3}$ nanosheets were prepared, and then its phototherapeutic properties were investigated. The bulk $\mathrm{FePS}_{3}$ was synthesized by a high-temperature solid-phase method and $\mathrm{FePS}_{3}$ nanosheets were obtained by an ultrasonic-assisted liquid phase exfoliating method. The nanosheets displayed particle morphology with average hydrated size of less than $200 \mathrm{~nm}$ (153 nm in average), performed photothermal conversion efficiency of $19.7 \%$ under $1064 \mathrm{~nm}$ laser irradiation, and generated reactive oxygen species under $660 \mathrm{~nm}$ laser irradiation. Cell experiment results showed that $\mathrm{FePS}_{3}$ nanosheets had excellent photothermal and photodynamic therapeutic effects. Therefore, $\mathrm{FePS}_{3}$ nanosheets can be used as both photothermal agent and photosensitizer to achieve combined
\end{abstract}

收稿日期：2020-12-31；收到修改稿日期：2021-02-26; 网络出版日期：2021-03-15

基金项目：上海理工大学科技发展项目(2018KJFZ016, 2019KJFZ023)

Project of University of Shanghai for Science and Technology (2018KJFZ016, 2019KJFZ023)

作者简介: 杨 功(1996-), 女, 硕士研究生. E-mail: yangmai77@163.com

YANG Mai(1996-), female, Master candidate. E-mail: yangmai77@163.com

通信作者：陈 雨，教授.E-mail: chenyuedu@shu.edu.cn; 朱钰方，教授.E-mail: zjf2412@163.com

CHEN Yu, professor. E-mail: chenyuedu@shu.edu.cn; ZHU Yufang, professor. E-mail: zjf2412@163.com 
photothermal-photodynamic therapy for treating tumors, which shows great application potential.

Key words: two-dimensional nanomaterial; $\mathrm{FePS}_{3}$ nanosheet; phototherapy; combined therapy

随着人们对光和光-物质相互作用的物理本质 了解得越来越深入, “生物医学光学”逐渐成为一个 新兴领域。光学治疗的快速发展催生了包括光热治 疗、光动力学治疗策略在内的肿瘤治疗手段。其中 光动力学治疗的三要素为光敏剂、一定波长的激光 刺激和组织氧。光动力学治疗可利用光化学反应产 生的单线态氧 $\left({ }^{1} \mathrm{O}_{2}\right)$ 等活性氧(Reactive oxygen species, ROS)杀死肿瘤细胞, 具有更小的侵入性、更低的副 作用和更弱的耐药性等优势 ${ }^{[1-3]}$ 。光热治疗则是利用 光热剂(Photothermal agent)将光能转化为热能, 产 生局部加热效果而消融血管化微环境较差的肿瘤。 光学治疗可以通过改变辐照部位、持续时间和激光 功率密度而精确控制治疗组织范围、时间和功效, 实现辐照区域可控, 确保更低的副作用和更好的能 量聚集，从而产生显著的疗效。但是，单一模式的光 学治疗不能完全消除顽固性肿瘤, 仍有肿瘤复发或 转移的风险。因此, 有必要探索设计包含至少一种 光学治疗方法的联合治疗策略。

在众多光敏剂和光热剂中, 拥有超薄结构的二 维(2D)纳米材料以其自身优异的光学特性、高比表 面积和独特的表面化学性质 ${ }^{[4-8]}$ 等特点, 在光学治 疗中显示出巨大的潜力。成功剥离石墨烯是 $2 \mathrm{D}$ 纳 米材料研究史上的一个里程碑 ${ }^{[9]}$, 由此开启了大量 $2 \mathrm{D}$ 纳米材料的探索。如 $2 \mathrm{D}$ 过渡金属碳化物、氮化 物和碳氮化物(MXenes) $)^{[10]}$ 、黑磷(BP)纳米片 ${ }^{[11]}$ 、过 渡金属二硫族元素化合物 (TMDs) ${ }^{[12]}$ 等, 并有一系 列 $2 \mathrm{D}$ 纳米材料应用于多功能纳米诊疗平台。最近, 一类新型的金属磷三硫族元素化合物 $\left(\mathrm{MPX}_{3}\right.$, 其中 $\mathrm{M}=\mathrm{Fe} 、 \mathrm{Mn} 、 \mathrm{Ni}$ 等, $\mathrm{X}=\mathrm{S}$ 或 $\mathrm{Se}$ ) $2 \mathrm{D}$ 材料受到关注。 $\mathrm{MPX}_{3}$ 具有范德华层状结构, 面内刚度 $\left(60 \sim 120 \mathrm{~N} \cdot \mathrm{m}^{-1}\right)$ 略高于石墨烯, 裂解能 $\left(0.29 \sim 0.54 \mathrm{~J} \cdot \mathrm{m}^{-2}\right)$ 则略低于石 墨烯, 易于从块状 $\mathrm{MPX}_{3}$ 剥离得到相应的纳米片 (Nanosheets, NSs $)^{[13-15]}$ 。Zhang 等 ${ }^{[13]}$ 采用理论计算得 到的 $2 \mathrm{D} \mathrm{MnPSe}_{3}$ 单层在室温下的载流子迁移率 $\left(625.9 \mathrm{~cm}^{2} \cdot \mathrm{V}^{-1} \cdot \mathrm{s}^{-1}\right)$ 高于其他典型的 $2 \mathrm{D}$ 材料, 表明光 催化过程中电子-空穴复合的可能性降低, 且理论 推测其导带和价带能级的位置跨越了水的氧化还原 电势, 有利于进行光催化水分解反应。 $\mathrm{Du}$ 等 ${ }^{[16]}$ 发现 块状 $\mathrm{MPX}_{3}$ 的带隙范围为 $1.3 \sim 3.5 \mathrm{eV}$, 恰可满足光催 化剂的可设计性, 能在光催化过程中更有效地利用 可见光。虽然 $\mathrm{MPX}_{3}$ 纳米材料的生物医学应用研究 才刚刚起步，但已展现出巨大的应用潜力 ${ }^{[17-18]}$ 。
Zhang 等 ${ }^{[17]}$ 基于 $\mathrm{FePS}_{3}$ 纳米片构建的纳米平台可用 于光热和化学动力学协同治疗肿瘤。Fang 等 ${ }^{\left[{ }^{[18]}\right.}$ 在 $\mathrm{FePSe}_{3}$ 纳米片外包裹癌细胞膜, 将其作为靶向癌细 胞的高效光热剂, 可联合免疫治疗, 进一步促进细 胞程序性死亡, 从而显著延长实验小鼠寿命。但 $\mathrm{FePS}_{3}$ 纳米片的光学治疗特性还有待探索。

本研究采用高温固相法合成了一种金属磷三硫 族元素化合物 $\mathrm{FePS}_{3}$ 块体, 并通过超声辅助的液相 剥离法制备了 $\mathrm{FePS}_{3}$ 纳米片 $\left(\mathrm{FePS}_{3} \mathrm{NSs}\right)$ 。利用其在 宽波长范围内的高效光吸收, 将其作为光热剂和光 敏剂, 构建具有光热治疗和光动力学治疗双功能的 联合治疗纳米体系, 即通过高热引发细胞坏死和引 入 $\operatorname{ROS}$ 诱导细胞调亡两种途径, 高效治疗肿瘤。

\section{1 实验方法}

\section{1 试剂}

铁 $(\geqslant 99 \%)$ 、块状红磷 $(99.999 \%)$ 、硫( $\geqslant 99.99 \%)$ 、 5,5-二甲基-1-吡咯啉- $N$-氧化物(DMPO， $\geqslant 98.0 \%$ )和 2,2,6,6-四甲基哌啶(TEMP， $\geqslant 99 \%$ 、 $N$-甲基吡咯烷 酮(NMP, $\geqslant 99.0 \%$ )和 1,3-二苯基异苯并呋喃(DPBF, 97\%)均购自默克化工技术(上海)有限公司。硫辛酸聚乙二醇聚合物(LA-PEG，MW:5000)购自上海亚亦 生物科技有限公司。DMEM 培养基、胎牛血清 (FBS)、青链霉素混合液、磷酸盐缓冲液(PBS)、Cell Counting Kit-8(CCK-8 试剂盒)和胰酶均购自上海 润成生物有限公司。Calcein-AM/PI 细胞活性与细胞 毒性检测试剂盒、活性氧检测试剂盒和线粒体膜电 位检测试剂盒 $(\mathrm{JC}-1)$ 均购自上海碧云天生物技术有 限公司。

\section{$1.2 \mathrm{FePS}_{3} \mathrm{NSs}$ 的制备及聚乙二醇修饰}

首先参照文献报道的高温固相合成法 ${ }^{[15]}$ 制备 $\mathrm{FePS}_{3}$ 块体。将对应于 $5 \mathrm{~g} \mathrm{FePS}_{3}$ 化学计量比的铁 $(\mathrm{Fe})$ 、 红磷 $(\mathrm{P})$ 和硫 $(\mathrm{S})$ 放置于石英玻璃管中, 用真空泵将 管内抽真空后, 使用氢氧焰焊机加热卡在管中部的 石英块, 使石英块与石英管壁相熔, 冷却后实现密 封。密封管在马弗炉中加热至 $700{ }^{\circ} \mathrm{C}$ 并保温 $6 \mathrm{~d}$, 得 到 $\mathrm{FePS}_{3}$ 块体。接着将块状 $\mathrm{FePS}_{3}$ 置于石英研钵中 充分研磨得到 $\mathrm{FePS}_{3}$ 粉末。取 $\mathrm{FePS}_{3}$ 粉末 $(0.3 \mathrm{~g})$ 置 于 $80 \mathrm{~mL} \mathrm{NMP}$ 中超声处理 $(600 \mathrm{~W}, 50 \mathrm{~h})$ 后离心 (3000 r/min, $30 \mathrm{~min}$ )去掉未剥离的大块材料, 最终 得到含有大量 $\mathrm{FePS}_{3}$ 纳米片的悬浮液。为获得良好 
的生物相容性, 选用 LA-PEG ${ }^{[19]}$ 对 $\mathrm{FePS}_{3}$ NSs 作进 一步修饰, 即将 $\mathrm{FePS}_{3} \mathrm{NSs}(20 \mathrm{mg})$ 和 LA-PEG (60 mg) 混合, 分散于去离子水中, 经 $30 \mathrm{~min}$ 超声分散后磁 性搅拌过夜, 最后离心并用去离子水洗涤数次, 以 去掉多余的 LA-PEG, 得到 PEG 修饰的 $\mathrm{FePS}_{3}$ $\mathrm{NSs}\left(\mathrm{FePS}_{3}-\mathrm{PEG}\right)$ 。

\section{3 光动力学效应测试}

活性氧检测试剂 DPBF 可以与 ${ }^{1} \mathrm{O}_{2}$ 发生 DielsAlder 1,4-环加成反应, 导致其在 $410 \mathrm{~nm}$ 处的光吸 收强度降低, 因此 DPBF 可作为 ${ }^{1} \mathrm{O}_{2}$ 探针用以检测 ${ }^{1} \mathrm{O}_{2}$ 。首先将含有 $\mathrm{FePS}_{3} \mathrm{NSs}(50 \mu \mathrm{g} / \mathrm{mL})$ 和 DPBF 的 混合液在黑暗中静置 $1 \mathrm{~h}$ 以达到吸附/解吸附的平衡 状态。随后用波长为 $660 \mathrm{~nm}$ 且功率密度为 $0.5 \mathrm{~W} / \mathrm{cm}^{2}$ 的激光辐照该混合液 $(3 \mathrm{~mL})$, 在不同时间点通过 UV-Vis-NIR 光谱仪(HORIBA FluoroMAX-4, France) 测定 $410 \mathrm{~nm}$ 处的吸光度。另外, 利用电子自旋共振 (ESR)光谱仪(Bruker EMX-8/2.7) 检测 ${ }^{1} \mathrm{O}_{2}$ 的产生, 将含有 ${ }^{1} \mathrm{O}_{2}$ 捕获剂 TEMP 的 $\mathrm{FePS}_{3} \mathrm{NSs}$ 悬浮液 $(50 \mu \mathrm{g} / \mathrm{mL})$ 暴露于激光 $\left(660 \mathrm{~nm}, 0.5 \mathrm{~W} / \mathrm{cm}^{2}\right)$ 下辐照 $10 \mathrm{~min}$, 然后将混合物迅速转移至标准毛细管中, 并立即记录 ESR 光谱。同样地, 使用羟自由基 $(\cdot \mathrm{OH})$ 或超氧阴离子 $\left(\mathrm{O}_{2}{ }^{-}{ }^{-}\right)$捕获剂 DMPO, 对比监测 $\mathrm{FePS}_{3}$ $\mathrm{NSs}$ 是否诱导产生其他自由基。

\section{4 光热性能测试}

利用 Fotric-225 热红外成像仪记录不同条件下 $\mathrm{FePS}_{3} \mathrm{NSs}$ 悬浮液的温度变化: (1)用 $1064 \mathrm{~nm}$ $\left(1 \mathrm{~W} / \mathrm{cm}^{2}\right)$ 激光分别辐照不同浓度 $(0 、 25 、 50 、 100$ 、 200 和 $300 \mu \mathrm{g} / \mathrm{mL}$ ) 的 $\mathrm{FePS}_{3} \mathrm{NSs} 10 \mathrm{~min}$; (2)用不同激 光功率密度的 $1064 \mathrm{~nm}$ 激光 $\left(0.5 、 1 、 1.5 、 2 \mathrm{~W} / \mathrm{cm}^{2}\right)$ 分别辐照 $\mathrm{FePS}_{3} \mathrm{NSs}(150 \mu \mathrm{g} / \mathrm{mL}) 10 \mathrm{~min}$; (3)用激光 功率密度为 $1 \mathrm{~W} / \mathrm{cm}^{2}$ 的 $1064 \mathrm{~nm}$ 激光辐照浓度为 $150 \mu \mathrm{g} / \mathrm{mL}$ 的 $\mathrm{FePS}_{3} \mathrm{NSs}$ 并进行 5 次激光“开一关”循 环辐照(共 $50 \mathrm{~min}$ )。另根据文献[20]报道的方法计算 得到 $\mathrm{FePS}_{3} \mathrm{NSs}(150 \mu \mathrm{g} / \mathrm{mL})$ 在 $1064 \mathrm{~nm}$ 光波 $\left(1 \mathrm{~W} / \mathrm{cm}^{2}\right)$ 处的激光光热转换系数。

\section{5 细胞毒性测试}

CCK-8 试剂盒中的水溶性四唑盐可与活细胞中 的脱氢酶发生氧化还原反应, 生成橙黄色甲珗化合 物且生成量与活细胞数量成正比。因此, 可利用酶 标仪读取此化合物的吸光度来计算细胞存活率。将 分散在 $100 \mu \mathrm{L}$ 培养基中的 $1 \times 10^{4} 4 \mathrm{~T} 1$ 小鼠乳腺癌细 胞(购自 ATCC)接种到 96 孔板中培养 $12 \mathrm{~h}$, 待细胞 贴壁后再分别与不同浓度的 $\mathrm{FePS}_{3}-\mathrm{PEG}(0 、 15 、 30$ 、 60、90、120 $\mu \mathrm{g} / \mathrm{mL}$ ) 共孵育 12 和 $24 \mathrm{~h}$ 。随后用溶解 在 DMEM 培养基中的 CCK-8 替代含有材料的培养
基以检测细胞毒性。

\section{6 $\mathrm{FePS}_{3}$-PEG 的体外抗肿瘤作用}

FePS $_{3}$-PEG 的光热消融肿瘤细胞效果: 将 $4 \mathrm{~T} 1$ 细胞 $\left(1 \times 10^{4} /\right.$ 孔)接种到 96 孔板中, 待细胞贴壁后分 别与不同浓度的 $\mathrm{FePS}_{3}-\mathrm{PEG}(0 、 25 、 50 、 100 \mu \mathrm{g} / \mathrm{mL})$ 共孵育 $4 \mathrm{~h}$ 以待材料被细胞内吞, 接着用 $1064 \mathrm{~nm}$ 激光 $\left(1 \mathrm{~W} / \mathrm{cm}^{2}\right)$ 辐照各孔细胞 $10 \mathrm{~min}$, 过夜培养后用 CCK-8 试剂盒方法评估细胞活力。

$\mathrm{FePS}_{3}$-PEG 的光动力学治疗抑制肿瘤细胞效 果: 将 $4 \mathrm{~T} 1$ 细胞 $\left(1 \times 10^{4} /\right.$ 孔 $)$ 接种到 96 孔板中, 待细 胞贴壁后与不同浓度的 $\mathrm{FePS}_{3}-\mathrm{PEG}(0 、 25 、 50$ 、 $100 \mu \mathrm{g} / \mathrm{mL}$ )共孵育 $4 \mathrm{~h}$, 随后用 $660 \mathrm{~nm}$ 激光 $\left(0.5 \mathrm{~W} / \mathrm{cm}^{2}\right)$ 辐照各孔细胞 $10 \mathrm{~min}$, 过夜培养后用 CCK-8 试剂盒 方法测定细胞活力。

$\mathrm{FePS}_{3}$-PEG 的光热和光动力学联合治疗效果: 利用 Calcein-AM(钲黄绿素)和 PI(碘化丙啶)两种苂 光探针可分别检测细胞内酯酶活性和细胞膜完整 性, 从而评价细胞活性与毒性。 $4 \mathrm{~T} 1$ 细胞 $\left(4 \times 10^{5} / \mathrm{III}\right)$ 在共聚焦血(底面孔 $\phi 14 \mathrm{~mm}$ )中培养贴壁后, 根据 以下分组作不同处理: (1)空白、(2) $\mathrm{FePS}_{3}-\mathrm{PEG}$ 、 (3) $1064 \mathrm{~nm}$ 、(4) $660 \mathrm{~nm} 、(5) \mathrm{FePS}_{3}-\mathrm{PEG}+1064 \mathrm{~nm}$ 、 (6) $\mathrm{FePS}_{3}-\mathrm{PEG}+660 \mathrm{~nm}$ 和(7) $\mathrm{FePS}_{3}-\mathrm{PEG}+1064 \mathrm{~nm}+$ $660 \mathrm{~nm}$ 。其中(3)和(5)组用功率密度 $0.5 \mathrm{~W} / \mathrm{cm}^{2}$ 的 $660 \mathrm{~nm}$ 激光辐照 $10 \mathrm{~min}$; (4)和(6)组用功率密度为 $1 \mathrm{~W} / \mathrm{cm}^{2}$ 的 $1064 \mathrm{~nm}$ 激光辐照 $10 \mathrm{~min}$; (7)组则用 $660 \mathrm{~nm}\left(0.5 \mathrm{~W} / \mathrm{cm}^{2}, 10 \mathrm{~min}\right)$ 和 $1064 \mathrm{~nm}\left(1 \mathrm{~W} / \mathrm{cm}^{2}\right.$, 10 min)激光先后辐照。最后经 Calcein-AM 和 PI 染 色 $15 \mathrm{~min}$, 用激光扫描共聚焦显微镜(CLSM)观察 评估细胞状态(观察活细胞: 激发光波长 $488 \mathrm{~nm}$, 发 射光波长 $515 \mathrm{~nm}$; 观察死细胞: 激发光波长 $535 \mathrm{~nm}$, 发射光波长 $617 \mathrm{~nm}$ )。

\section{7 细胞内活性氧检测}

细胞内的 ROS 可将被胞吞入细胞内的无苂光 的 2,7-二氯苂光素双乙酸酯(DCFH-DA) 氧化为有苂 光的 2,7-二氯荧光素, 从而通过荧光来检测 $\operatorname{ROS}$ 水 平。将 $4 \mathrm{~T} 1$ 细胞 $\left(4 \times 10^{5} / \mathrm{Il}\right.$ ) 接种在共聚焦血(底面孔 $\phi 14 \mathrm{~mm}$ )内培养, 分组为: (1)空白、(2) $\mathrm{FePS}_{3}-\mathrm{PEG、}$ (3) $1064 \mathrm{~nm}\left(1 \mathrm{~W} / \mathrm{cm}^{2}, 10 \mathrm{~min}\right) 、$ (4) $660 \mathrm{~nm}\left(0.5 \mathrm{~W} / \mathrm{cm}^{2}\right.$, $10 \mathrm{~min}) 、(5) \mathrm{FePS}_{3}-\mathrm{PEG}+1064 \mathrm{~nm}\left(1 \mathrm{~W} / \mathrm{cm}^{2}, 10 \mathrm{~min}\right) 、$ (6) $\mathrm{FePS}_{3}-\mathrm{PEG}+660 \mathrm{~nm}\left(0.5 \mathrm{~W} / \mathrm{cm}^{2}, 10 \mathrm{~min}\right)$ 和 (7) $\mathrm{FePS}_{3}-\mathrm{PEG}+1064 \mathrm{~nm}\left(1 \mathrm{~W} / \mathrm{cm}^{2}, 10 \mathrm{~min}\right)+660 \mathrm{~nm}$ $\left(0.5 \mathrm{~W} / \mathrm{cm}^{2}, 10 \mathrm{~min}\right)$ 。待细胞贴壁后将(2)、(5)、(6)、 (7)组与材料共孵育 $4 \mathrm{~h}$, 之后所有组用 DCFH-DA 染色 $30 \mathrm{~min}$ 再进行光照刺激, 最后在 CLSM 下观察 ROS 的 生成情况(激发光波长 $480 \mathrm{~nm}$, 发射光波长 $525 \mathrm{~nm}$ )。 


\section{8 细胞内线粒体膜电位变化检测}

JC-1 (5,5',6,6'-tetrachloro-1, 1', 3,3'-tetraethylbenzimidazolyl-carbocyanine iodide)分子会在膜电位 较高的线粒体基质内形成发红色苂光的聚合物, 在 相反情况下以发绿色荧光的单体形式存在，由此可 捕捉到细胞早期凋亡的标志之一: 线粒体膜电位下 降。先在共聚焦皿(底面孔 $\phi 14 \mathrm{~mm}$ )内培养 $4 \mathrm{~T} 1$ 细胞 $\left(4 \times 10^{5} /\right.$ 皿) 至其贴壁, 根据分组对细胞进行相应处 理: (1)空白、(2) FePS 3 -PEG、(3) $1064 \mathrm{~nm}\left(1 \mathrm{~W} / \mathrm{cm}^{2}\right.$, $10 \mathrm{~min}) 、(4) 660 \mathrm{~nm}\left(0.5 \mathrm{~W} / \mathrm{cm}^{2}, 10 \mathrm{~min}\right) 、(5)$ $\mathrm{FePS}_{3}-\mathrm{PEG}+1064 \mathrm{~nm} \quad\left(1 \mathrm{~W} / \mathrm{cm}^{2}, \quad 10 \mathrm{~min}\right)$ 、 (6) $\mathrm{FePS}_{3}-\mathrm{PEG}+660 \mathrm{~nm}\left(0.5 \mathrm{~W} / \mathrm{cm}^{2}, 10 \mathrm{~min}\right)$ 和 (7) $\mathrm{FePS}_{3}-\mathrm{PEG}+1064 \mathrm{~nm}\left(1 \mathrm{~W} / \mathrm{cm}^{2}, 10 \mathrm{~min}\right)+660 \mathrm{~nm}$ $\left(0.5 \mathrm{~W} / \mathrm{cm}^{2}, 10 \mathrm{~min}\right)$ 。即(2)、(5)、(6)、(7)组与 $\mathrm{FePS}_{3}$-PEG 共孵育 $4 \mathrm{~h}$ 后, 用 $660 \mathrm{~nm}$ 激光辐照(4)和 (6)组; (3)和(5)组用 $1064 \mathrm{~nm}$ 激光辐照; (7)组用两种 波长激光先后辐照。随后对所有组的细胞作 JC-1 染 色 $30 \mathrm{~min}$, 最后用 CLSM 观察线粒体膜电位变化(激 发光波长 $480 \mathrm{~nm}$, 发射光波长 $525 \mathrm{~nm}$ )。

\section{2 结果与讨论}

\section{1 $\mathrm{FePS}_{3} \mathrm{NSs}$ 的制备与表征}

采用文献报道的高温固相合成法一步烊烧各元 素粉末得到大块的 $\mathrm{FePS}_{3}$ 晶体 ${ }^{[15]}$ 。通过 XRD 分析 可知(图 1(a)), 所得 $\mathrm{FePS}_{3}$ 固体的各衍射峰与 $\mathrm{FePS}_{3}$ 标准卡片(PDF\#30-0663)基本一致, $2 \theta=13.8^{\circ}$ 和 $27.8^{\circ}$ 处的强衍射峰分别对应于 $\mathrm{FePS}_{3}$ 晶体的(001)和(002) 晶面, 表明本实验成功合成了具有较高相纯度的结 晶 $\mathrm{FePS}_{3}$ 块体。从 $\mathrm{FePS}_{3}$ 块体的扫描电镜 $(\mathrm{SEM})$ 照片 (图 1(b)) 可观察到独特的层状堆叠结构, 说明该材 料具有从块体剥离得到相应纳米片的可能。

进一步地，采用超声辅助液相剥离法 ${ }^{[21]}$ 对高温 合成的 $\mathrm{FePS}_{3}$ 块体进行剥离, 得到 $\mathrm{FePS}_{3} \mathrm{NSs}$ (图 2(a))。
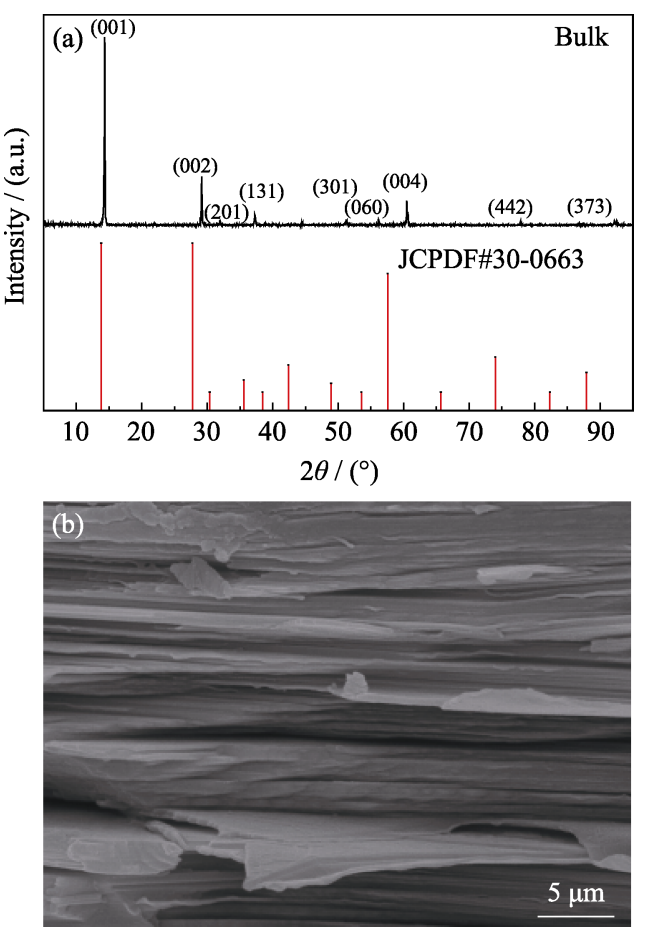

图 $1 \mathrm{FePS}_{3}$ 块体的 $\mathrm{X}$ 射线衍射图谱(a)和扫描电镜照片(b)

Fig. 1 XRD pattern (a) and SEM image (b) of bulk FePS

动态光散射测得 LA-PEG 修饰前后的纳米片平均水 合粒径分别为 153 (图 2(b)) 和 $176 \mathrm{~nm}$ (图 2(c))。 LA 修饰的 PEG (LA-PEG)在 PEG 末端具有一个二硫基, 通过硫醇反应可功能化过渡金属硫化物 ${ }^{[22]}$ 。将 PEG 修饰后的 $\mathrm{FePS}_{3}-\mathrm{PEG}$ 分别分散于 PBS 和细胞培养液 DMEM 中, $24 \mathrm{~h}$ 后材料未出现明显聚集沉降(图 2(c) 插图), 说明其在 PBS 和 DMEM 中具有良好的分散 性，有利于提高其细胞相容性。

\section{$2.2 \mathrm{FePS}_{3} \mathrm{NSs}$ 的光动力学效应}

$\mathrm{FePS}_{3} \mathrm{NSs}$ 的 UV-Vis-NIR 漫反射光谱及相应的 Tauc 图(图 3 和插图)。根据 Kubelka-Munk 理论, 吸收 系数 $(\alpha)$ 可以通过测量漫反射率(图 3)并代入 KubelkaMunk 函数 $(F(R))$ 计算获得 ${ }^{[23]}$, 因为 $\mathrm{FePS}_{3}$ 是一种间 接带隙半导体, 将 $(\alpha h v)^{1 / 2}$ 设为 Tauc 图的 $Y$ 轴 ${ }^{[24]}$ 。由
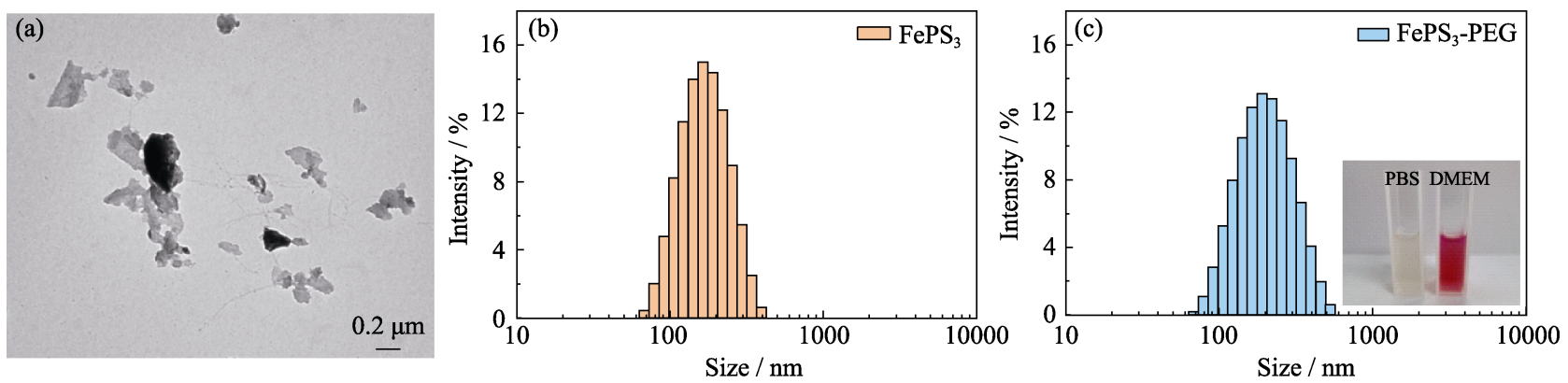

图 $2 \mathrm{FePS}_{3} \mathrm{NSs}$ 的透射电镜照片(a), PEG 修饰前(b)和修饰后(c)的平均水合粒径及其分散于 PBS 和 DMEM 中的照片(插图)

Fig. 2 TEM image (a) of $\mathrm{FePS}_{3}$ nanosheets (NSs) and hydrodynamic size of $\mathrm{FePS}_{3}$ nanosheets (NSs) before (b) and after (c) PEGylation with inset showing the picture of $\mathrm{FePS}_{3}$-PEG dispersed in PBS and in DMEM 


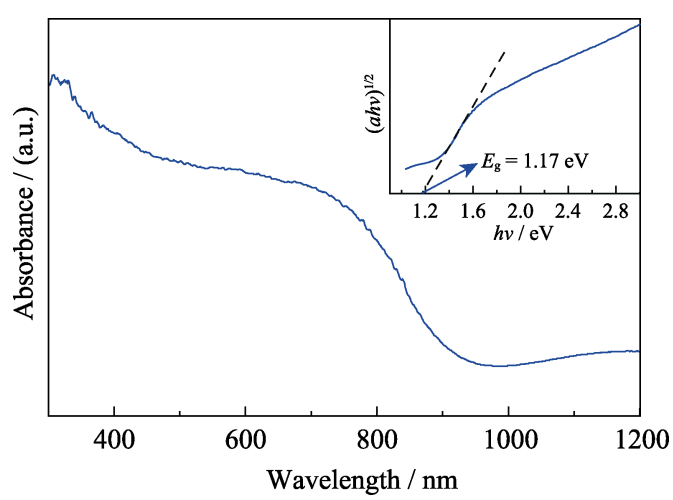

图 $3 \mathrm{FePS}_{3} \mathrm{NSs}$ 的 UV-Vis-NIR 漫反射光谱和估算的带隙 电势(插图)

Fig. 3 UV-Vis-NIR diffuse reflectance spectrum of $\mathrm{FePS}_{3} \mathrm{NSs}$ with inset showing the estimated band gap potential

Tauc 图中切线与 $X$ 轴 $(h v)$ 的截距, 估算得到该 $\mathrm{FePS}_{3}$ $\mathrm{NSs}$ 的带隙值 $\left(E_{\mathrm{g}}\right)$ 为 $1.17 \mathrm{eV}^{[25]}$ (图 3 插图), 说明 $\lambda \leqslant 1059 \mathrm{~nm}$ 的激光可满足其电子跃迁到激发态所 需要吸收的光子能量。

$\mathrm{FePS}_{3} \mathrm{NS}$ 在 600 800 nm 范围内具有较强的光 吸收性能, 且在该范围内的光穿透深度可达 4 $8 \mathrm{~mm}^{[26]}$, 同时光的波长仍然具有足够的能量以产 生单线态氧 ${ }^{[27]}$ 。因此, 该 $\mathrm{FePS}_{3} \mathrm{NSs}$ 可以通过 $660 \mathrm{~nm}$ 激光辐照获取分离的光致电子来实现电子-空穴的 空间分离, 而该电子可以与环境中的 $\mathrm{O}_{2}$ 反应生成 $\operatorname{ROS}^{[28]}$ 。

以 DPBF 作为探针检测混合液中 $\mathrm{FePS}_{3} \mathrm{NSs}$ 受 激光激发 $\mathrm{O}_{2}$ 生成 ${ }^{1} \mathrm{O}_{2}$ 的情况。如图 4(a)所示, 存在 $\mathrm{FePS}_{3} \mathrm{NSs}$ 时, 经过 $660 \mathrm{~nm}$ 激光 $\left(0.5 \mathrm{~W} / \mathrm{cm}^{2}\right)$ 辐照, $410 \mathrm{~nm}$ 处的吸光度随辐照时间延长而不断衰减, 这 表明 DPBF 被生成的 ${ }^{1} \mathrm{O}_{2}$ 氧化。此现象与仅含 DPBF 的溶液形成鲜明对比(图 4(b)), 其特征峰吸光度在 激光辐照下变化微弱。

另外, 为获得在 $660 \mathrm{~nm}$ 激光辐照下由 $\mathrm{FePS}_{3}$
NSs 产生 ROS 的直接证据, 本研究还作了电子自旋 共振光谱分析。将 TEMP 和 DMPO 分别用作 ${ }^{1} \mathrm{O}_{2}$ 和 $\cdot \mathrm{OH}\left(\right.$ 或 $\mathrm{O}_{2}{ }^{-}$) 捕获剂。只有在激光辐照下才能观察 到具有 $1: 1: 1$ 的三重态光谱的 ${ }^{1} \mathrm{O}_{2}$ 信号特征, 但未 检测到. $\mathrm{OH}$ 和 $\mathrm{O}_{2}{ }^{-}$信号(图 4(c)), 表明 $\mathrm{FePS}_{3} \mathrm{NSs}$ 可 将光诱导的能量从其自身 (光敏剂)转移到基态氧 $\left({ }^{3} \mathrm{O}_{2}\right)$, 并产生具有细胞毒性的 ${ }^{1} \mathrm{O}_{2}{ }^{[29]}$ 。

\section{$2.3 \mathrm{FePS}_{3} \mathrm{NSs}$ 的光热性能}

不同浓度 $\mathrm{FePS}_{3} \mathrm{NSs}$ 的 Vis-NIR 吸收光谱图 显出该材料在近红外一区 $(750 \sim 1000 \mathrm{~nm})$ 和二区 $(1000 \sim 1200 \mathrm{~nm})$ 有较强的光吸收能力, 如图 5(a) 所示。

由于近红外二区激光具有更低的人体组织背景 和更弱的光子散射, 在光热治疗中能产生更有效的 组织穿透效果 ${ }^{[30-31]}$, 且具有更高的激光最大允许曝 光量(Maximum permissible exposure)。又因 $\mathrm{FePS}_{3}$ NSs 在近红外二区生物耐受剂量范围中具有优异的 光吸收能力, 本研究评估了其在 $1064 \mathrm{~nm}$ 处的光热 转换性能。

首先, 在 $1064 \mathrm{~nm}$ 波长的激光辐照下, $\mathrm{FePS}_{3} \mathrm{NSs}$ 表现出随材料浓度或激光功率密度增加而显著增强 的升温效果(图 5(b, c))。在 $1064 \mathrm{~nm}$ 激光 $\left(1 \mathrm{~W} / \mathrm{cm}^{2}\right)$ 辐照 $10 \mathrm{~min}$ 时, $\mathrm{FePS}_{3} \mathrm{NSs}$ 悬浮液 $(300 \mu \mathrm{g} / \mathrm{mL})$ 的温 度升高了 $21{ }^{\circ} \mathrm{C}$, 而在相同的激光功率密度下, 去 离子水只观察到微弱的升温现象。通过增大辐射功 率密度可增强光热性能(图 5(c)), 进一步证明了该 纳米片在光热转化中的可调节能力。另外, 用热红 外成像仪实时记录的红外热成像图也直观地呈现了 不同浓度 $\mathrm{FePS}_{3} \mathrm{NSs}$ 在 $1064 \mathrm{~nm}$ 激光 $\left(1 \mathrm{~W} / \mathrm{cm}^{2}\right)$ 辐照 $10 \mathrm{~min}$ 内的产热能力(图 6)。

此外, 如图 5(d)所示, 在 5 次激光开/关辐照循环 中观察到 $\mathrm{FePS}_{3} \mathrm{NSs}$ 的温度变化情况基本相同, 且
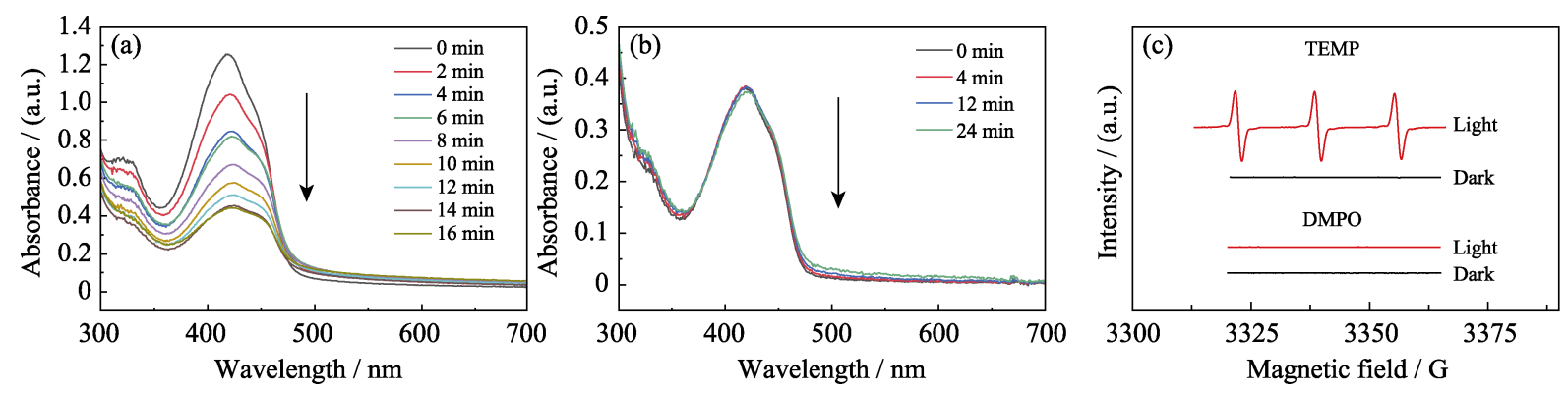

图 $4660 \mathrm{~nm}$ 激光照射下 $\mathrm{FePS}_{3} \mathrm{NSs}$ 与 DPBF 的混合液(a)和纯 DPBF 溶液(b)的紫外-可见吸收光谱,

以及不同反应体系的 ESR 光谱图(c)

Fig. 4 UV-Vis absorption spectra of the mixture solution of $\mathrm{FePS}_{3} \mathrm{NSs}$ mixed with DPBF (1,3-diphenylisobenzofuran) (a) and DPBF solution (b) under $660 \mathrm{~nm}$ laser irradiation, and ESR spectra of different reaction systems (c) TEMP: a reagent used to detect ${ }^{1} \mathrm{O}_{2}$. DMPO: a reagent used to detect $\mathrm{O}_{2}{ }^{-{ }^{-}}$and $\cdot \mathrm{OH}$ 

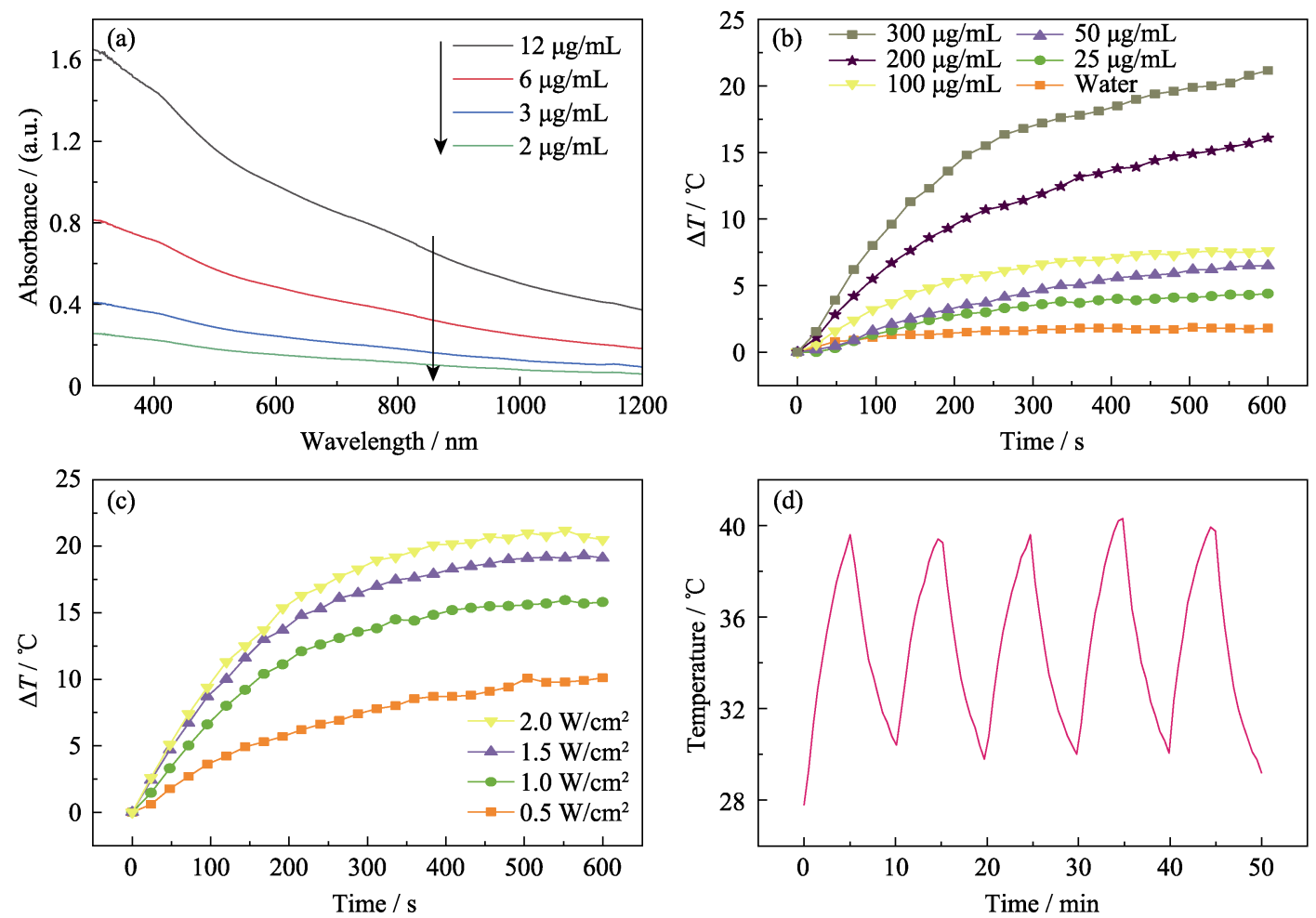

图 5 不同浓度 $\mathrm{FePS}_{3} \mathrm{NSs}$ 的可见-近红外吸收光谱图(a), 不同浓度(b)和不同激光功率密度 $(1064 \mathrm{~nm}$ 激光)(c) 条件下 $\mathrm{FePS}_{3} \mathrm{NSs}$ 随时间的光热升温曲线, 以及 $\mathrm{FePS}_{3} \mathrm{NSs} 5$ 次激光开闭循环辐照的温度曲线(d)

Fig. 5 Vis-NIR spectra of $\mathrm{FePS}_{3}$ NSs with different concentrations (a), photothermal heating curves for different time at different concentrations (b), and different laser power densities (1064 nm laser) (c), and photothermal curve of FePS ${ }_{3}$ NSs under 5 cycles of laser "on-off" (d)

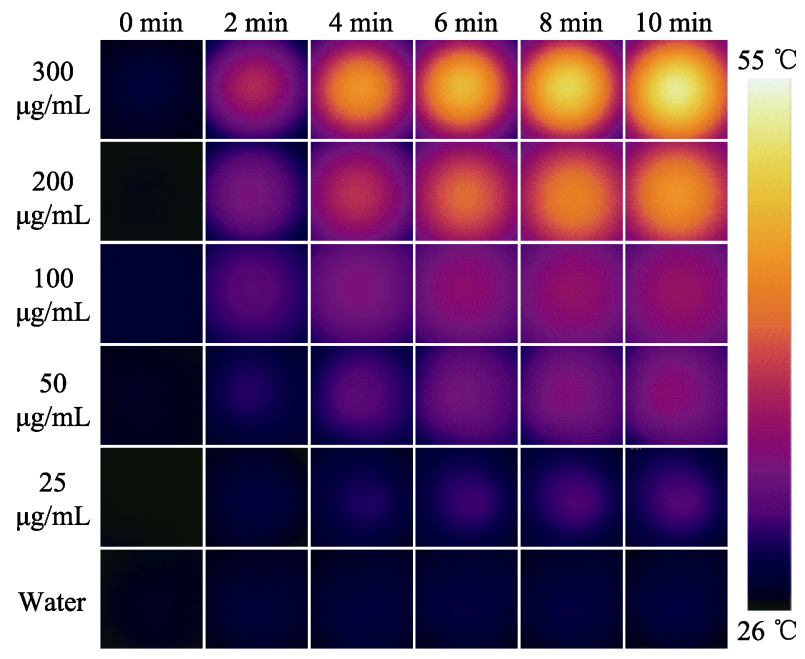

图 6 不同浓度 $\mathrm{FePS}_{3} \mathrm{NSs}$ 在 $1064 \mathrm{~nm}$ 激光照射下随时间升 温的红外热成像照片

Fig. 6 Thermal images of different concentrations of $\mathrm{FePS}_{3}$ nanosheets heated by $1064 \mathrm{~nm}$ laser irradiation for different time

升温能力并未逐渐减弱, 证明 $\mathrm{FePS}_{3} \mathrm{NSs}$ 具有良好 的光热稳定性。如图 7 所示, 根据兰伯特-比尔定律 计算, $\mathrm{FePS}_{3} \mathrm{NSs}$ 在 $1064 \mathrm{~nm}$ 光波处的消光系数 $(\varepsilon)$ 为 $38.68 \mathrm{~L} \cdot \mathrm{g}^{-1} \cdot \mathrm{cm}^{-1}$ (图 7(a)) $)^{[20]}$, 显著高于氧化石墨烯 $(\mathrm{GO})$ 纳米片 $\left(3.6 \mathrm{~L} \cdot \mathrm{g}^{-1} \cdot \mathrm{cm}^{-1}\right)^{[32]}$ 和 $\mathrm{WS}_{2}$ 纳米片 $\left(23.8 \mathrm{~L} \cdot \mathrm{g}^{-1} \cdot \mathrm{cm}^{-1}\right)^{[19]}$,
表明 $\mathrm{FePS}_{3} \mathrm{NSs}$ 具有较强的近红外激光吸收性能, 可用作光热转换的理想光热剂。随后，根据传热时 间常数 $\tau_{\mathrm{s}}=138.04$ (图 7(b)) 和最高稳态温度计算, $\mathrm{FePS}_{3} \mathrm{NSs}$ 的光热转换效率 $(\eta)$ 为 $19.7 \%$ (图 7(c)), 高于 已有报道的铜纳米线 $(12.5 \%)^{[33]}$ 和金纳米壳 $(13 \%)^{[34]}$ 。

\section{4 材料的细胞毒性及光学治疗效果}

本研究利用 CCK-8 分析评估了 $\mathrm{FePS}_{3}$-PEG 的体 外细胞毒性。如图 8 所示, $4 \mathrm{~T} 1$ 细胞与不同浓度 $\mathrm{FePS}_{3}-\mathrm{PEG}$ 纳米片在黑暗中分别共孵育 12 和 $24 \mathrm{~h}$ 后, 细胞活力并未受到明显抑制, 且与 $120 \mu \mathrm{g} / \mathrm{mL}$ 的 $\mathrm{FePS}_{3}$-PEG 共孵育 $24 \mathrm{~h}$ 后还能保持 $94.7 \%$ 的细胞 活力, 表明材料在检测浓度范围内具有良好的细胞 相容性。

为了研究 $\mathrm{FePS}_{3}-\mathrm{PEG}$ 纳米片的光学治疗作用, 本研究将 $4 \mathrm{~T} 1$ 细胞与安全范围内的不同浓度 $\mathrm{FePS}_{3}$-PEG 共孵育 $4 \mathrm{~h}$ 后分别用 $660 \mathrm{~nm}\left(0.5 \mathrm{~W} / \mathrm{cm}^{2}\right.$, $10 \mathrm{~min})$ 和 $1064 \mathrm{~nm}\left(1 \mathrm{~W} / \mathrm{cm}^{2}, 10 \mathrm{~min}\right)$ 激光辐照进行 光动力学治疗和光热治疗 (图 9), 当 $4 \mathrm{~T} 1$ 细胞经光动 力学治疗或者光热治疗后, 细胞活力明显降低。当材 料浓度为 $100 \mu \mathrm{g} / \mathrm{mL}$ 时, 单独经 $660 \mathrm{~nm}$ 激光辐照后 (光动力学治疗)的细胞活力仅为 $25 \%$ (图 9(a)); 类 似地, 单独经 $1064 \mathrm{~nm}$ 激光照射后(光热治疗), 细 

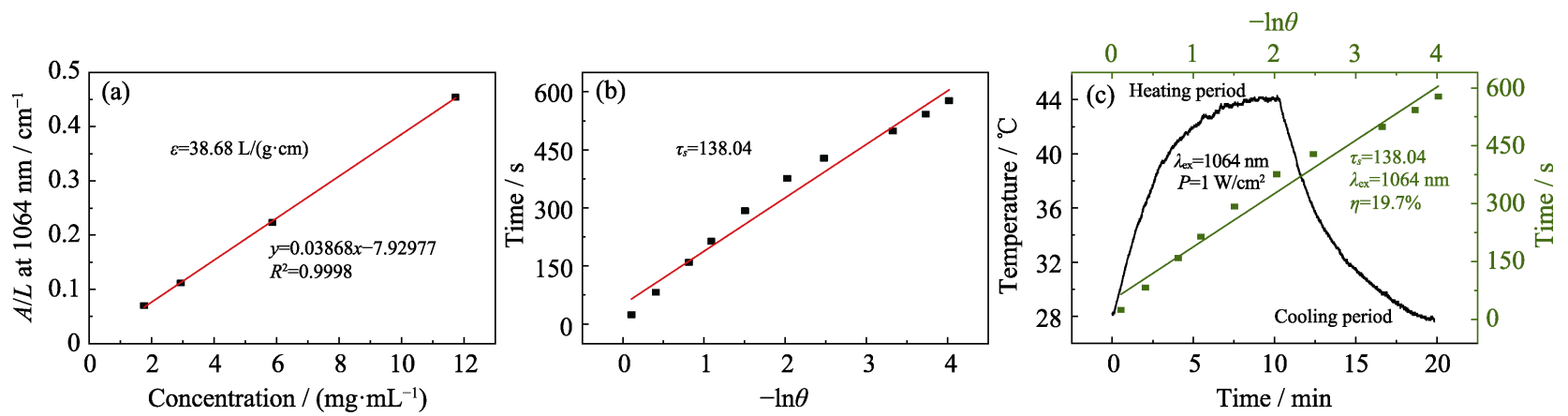

图 $7 \mathrm{FePS}_{3} \mathrm{NSs}$ 在 $\lambda=1064 \mathrm{~nm}$ 处的归一化吸收强度除以相应浓度下样品特征长度 $(A / L)$ 与相应浓度的线性拟合曲线(a), $\mathrm{FePS}_{3} \mathrm{NSs}$ 经 $1064 \mathrm{~nm}$ 激光辐照后冷却过程的 $-\ln \theta$ 与时间的线性关系(b), FePS $\mathrm{NSs}_{3}$ 在 $1064 \mathrm{~nm}$ 激光辐照下的升温和冷却曲线及冷却过程的 $-\ln \theta$ 与时间的线性关系(c)

Fig. 7 Linear fitting curve between normalized absorption intensity of $\mathrm{FePS}_{3} \mathrm{NSs}$ at $\lambda=1064 \mathrm{~nm}$ divided by the characteristic length of the sample at corresponding concentration $(A / L)$ and the corresponding concentration (a), linear relationship between $-\ln \theta$ and time of cooling process of $\mathrm{FePS}_{3}$ NSs after $1064 \mathrm{~nm}$ laser irradiation (b), heating and cooling curves of FePS $\mathrm{NSs}_{3}$ under $1064 \mathrm{~nm}$ laser irradiation, and linear relationship between $-\ln \theta$ and time of the cooling process (c) $\varepsilon, \tau_{\mathrm{s}}$ and $\eta$ represent extinction coefficient, time constant in cooling stage, and photothermal conversion efficiency, respectively, of $\mathrm{FePS}_{3} \mathrm{NSs}$ under $1064 \mathrm{~nm}$ laser irradiation

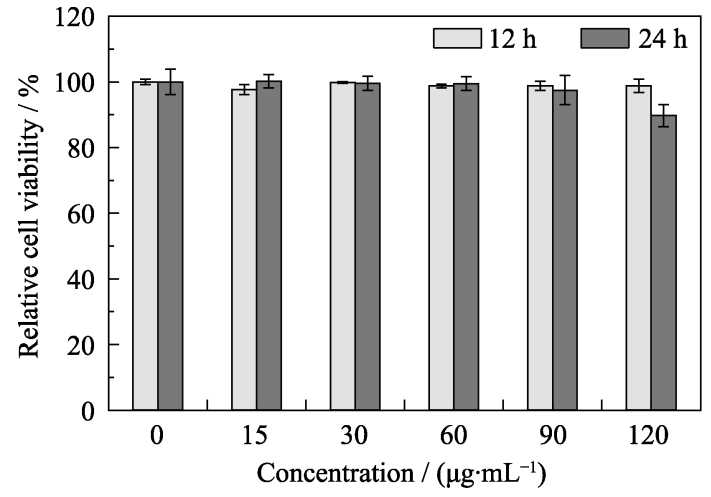

图 $84 \mathrm{~T} 1$ 细胞与不同浓度 $\mathrm{FePS}_{3}-\mathrm{PEG}$ 共孵育后的相对活力

Fig. 8 Relative cell viabilities of $4 \mathrm{~T} 1$ cells after incubation with different concentrations of $\mathrm{FePS}_{3}$-PEG

胞活力仅为 $13 \%$ (图 9(b)), 即细胞致死率高达 $87 \%$ 。 光动力学治疗和光热治疗均表现出随 $\mathrm{FePS}_{3}-\mathrm{PEG}$ 浓 度增加而增强的 $4 \mathrm{~T} 1$ 细胞生长抑制效果。

此外, CLSM 观察结果也证实了激光辐照下 $\mathrm{FePS}_{3}-\mathrm{PEG}$ 显著的光毒性(图 10(a))。与对照组相比, 单纯的材料或激光辐照处理对细胞活力几乎没有影 响，而在材料用 660 或 $1064 \mathrm{~nm}$ 激光辐照组观察到 部分死细胞(红色), 而联合治疗组则表现出最明显 的 4T1 细胞杀伤效果。另外, 使用 DCFH-DA 探针 可以检测光动力学效应中 ROS 的产生。如图 10(b) 所示, 只有同时满足存在 $\mathrm{FePS}_{3}$-PEG 且被 $660 \mathrm{~nm}$ 激 发这两个条件的单纯光动力学治疗组和联合治疗组 才能观察到指示 ROS 的绿色荧光, 表明 $\mathrm{FePS}_{3}-\mathrm{PEG}$ 可在激光辐照下产生电子-空穴空间分离, 并与周 围水或 $\mathrm{O}_{2}$ 分子进一步相互作用生成 ROS。过量的 ROS 会导致线粒体膜通透性增加和线粒体膜电位 丧失，释放调亡因子并最终诱导细胞调 [ $^{[35-36]}$ 。
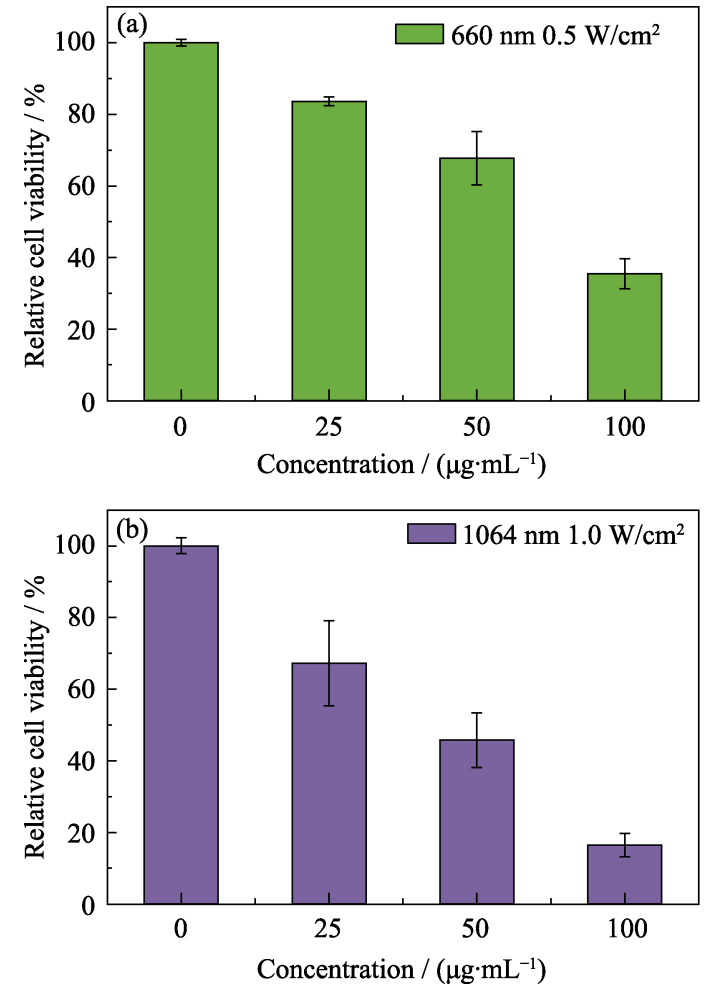

图 9 与不同浓度 $\mathrm{FePS}_{3}-\mathrm{PEG}$ 共孵育后对 $4 \mathrm{~T} 1$ 细胞进行的 体外光动力学治疗 (a) 和光热治疗 (b)

Fig. 9 In vitro photodynamic therapy (a) and photothermal therapy (b) treatment of 4T1 cells after incubation with different concentrations of $\mathrm{FePS}_{3}$-PEG

JC-1 作为一种亲脂性阳离子染料, 可以选择性地进 入线粒体，在进入正常生理功能的线粒体后，JC-1 转化为红色的聚集体, 而在功能异常的线粒体中则 表现为绿色的单体。如图 10(c)所示，与材料共孵育 且经 $660 \mathrm{~nm}$ 激光辐照组观察到显著的线粒体损伤, 说明 $\mathrm{FePS}_{3}-\mathrm{PEG}$ 可作为光敏剂生成大量 ROS, 使线 

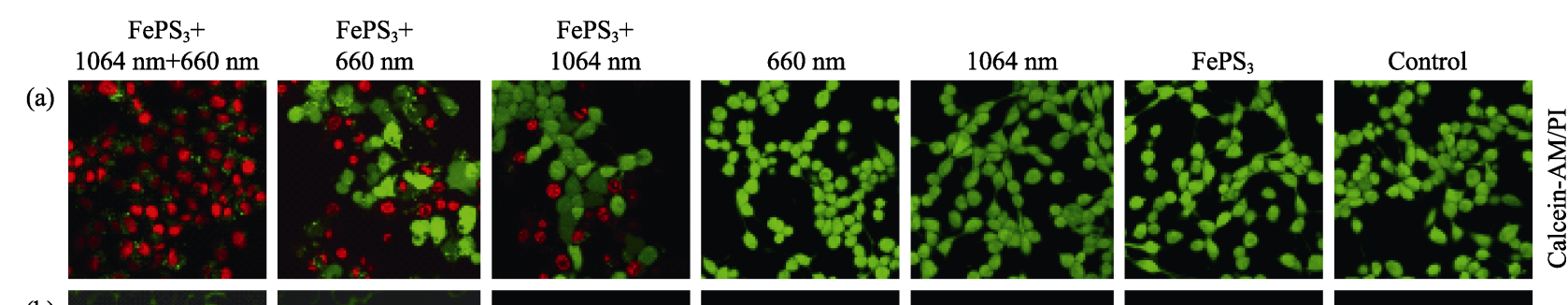

(b)
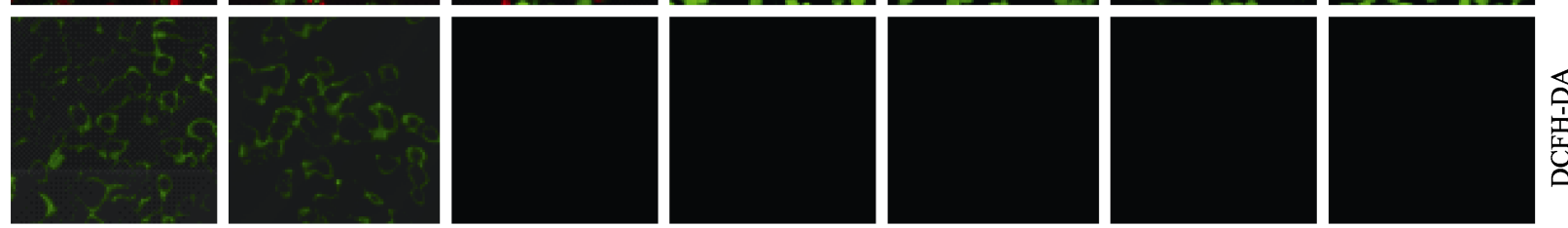

(c)
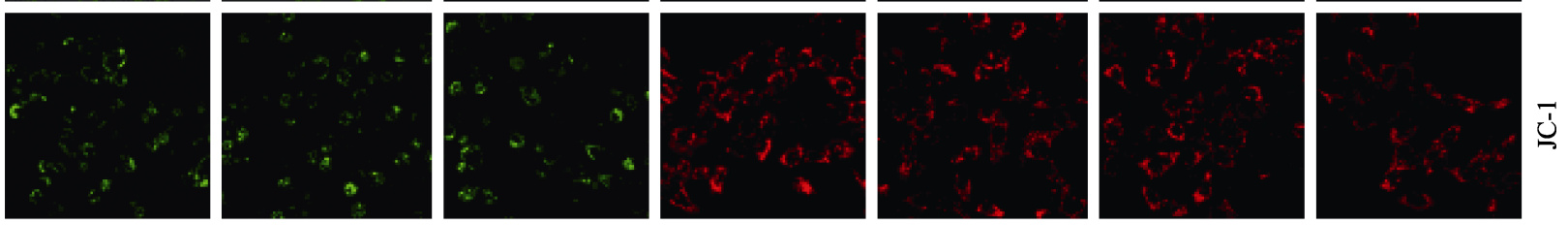

图 10 经不同处理后的 $4 \mathrm{~T} 1$ 细胞活力(a)、ROS 产物(b)及线粒体膜电位变化(c)的 CLSM 苂光照片

Fig. 10 Confocal laser scanning microscope (CLSM) images of 4T1 cell viabilities (a), reactive oxygen species

(ROS) production (b) and changes in mitochondrial membrane potential (c) after different treatments Calcein-AM/PI, DCFH-DA and JC-1 represent methods for detecting cell viability, ROS production, and changes in mitochondrial membrane potential, respectively

粒体功能失调并最终诱导癌细胞凋亡。另外由于线 粒体对热敏感, 单纯光热治疗组中近红外光辐照引 起的细胞内温度升高也会导致线粒体膜的通透性增

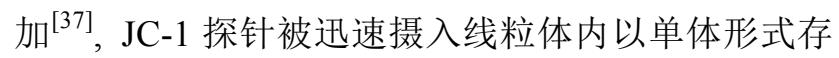
在并发出绿色荧光。说明光热治疗过程中激光辐照 产生的局部高温也能导致肿瘤细胞凋亡。

\section{3 结论}

本研究采用高温固相合成法㷽烧元素粉末得 到 $\mathrm{FePS}_{3}$ 块状晶体, 对块状晶体材料进行超声液相 剥离, 离心收集得到具有二维片状结构且平均水合 粒径约为 $153 \mathrm{~nm}$ 的 $\mathrm{FePS}_{3}$ 纳米片。LA-PEG 修饰 $\mathrm{FePS}_{3}$ 纳米片可提高其细胞相容性和稳定性。FePS 3 在可见光区域的光激发特性和在近红外二区生物耐 受剂量范围内显著的辐射吸收性能, 赋予其肿瘤光 学治疗功能。 $\mathrm{FePS}_{3}$ 纳米片在 $660 \mathrm{~nm}$ 激光辐照下能 将基态氧转化为 ${ }^{1} \mathrm{O}_{2}$ 而诱导细胞调亡, 获得光动力 学治疗效果; 在 $1064 \mathrm{~nm}$ 激光辐照下可将光能转换 为热能并实现 $19.7 \%$ 的光热转化效率, 获得光热治 疗效果。最为重要的是, $\mathrm{FePS}_{3}$ 纳米片可以实现光热 和光动力学联合治疗肿瘤效果。因此, 本研究制备 的 $\mathrm{FePS}_{3}$ 纳米片在肿瘤治疗领域具有较大的应用 潜力。

\section{参考文献:}

[1] LOVELL J F, LIU T W B, CHEN JUAN, et al. Activatable photosensitizers for imaging and therapy. Chemical Reviews, 2010, 110(5): 2839-2857.

[2] CELLI J P, SPRING B Q, RIZVI I, et al. Imaging and photodynamic therapy: mechanisms, monitoring, and optimization. Chemical Reviews, 2010, 110(5): 2795-2838.

[3] DOLMANS D E J G J, FUKUMURA D, JAIN R K. Photodynamic therapy for cancer. Nature Reviews Cancer, 2003, 3(5): 380-387.

[4] PENG B, ANG P K, LOH K P. Two-dimensional dichalcogenides for light-harvesting applications. Nano Today, 2015, 10(2): 128-137.

[5] LI L, KIM J, JIN C, et al. Direct observation of the layer-dependent electronic structure in phosphorene. Nature Nanotechnology, 2016, 12: $21-25$.

[6] LI XUAN-HUA, ZHU JIN-MENG, WEI BING-QING. Hybrid nanostructures of metal/two-dimensional nanomaterials for plasmonenhanced applications. Chemical Society Reviews, 2016, 45: 3145-3187.

[7] NOVOSELOV K S, GEIM A K, MOROZOV S V, et al. Electric field effect in atomically thin carbon films. Science, 2004, 306(5696): 666-669.

[8] CHHOWALla M, LIU ZHONG-FAN, ZHANG HUA. Twodimensional transition metal dichalcogenide (TMD) nanosheets. Chemical Society Reviews, 2015, 44(9): 2584-2586.

[9] ALLEN M J, TUNG V C, KANER R B. Honeycomb carbon: a review of graphene. Chemical Reviews, 2009, 110(1): 132-145.

[10] NAGUIB M, KURTOGLU M, PRESSER V, et al. Two-dimensional nanocrystals produced by exfoliation of $\mathrm{Ti}_{3} \mathrm{AlC}_{2}$. Advanced Materials, 2011, 23(37): 4248-4253.

[11] LIU HAN, NEAL A T, ZHU ZHEN, et al. Phosphorene: a new 2D material with high carrier mobility. ACS Nano, 2014, 8(4): 4033-4041.

[12] MANZELI S, OVCHINNIKOV D, PASQUIER D, et al. 2D transition metal dichalcogenides. Nature Reviews Materials, 2017, 2: 17033.

[13] ZHANG XU, ZHAO XU-DONG, WU DI-HUA, et al. $\mathrm{MnPSe}_{3}$ monolayer: a promising $2 \mathrm{D}$ visible-light photohydrolytic catalyst with high carrier mobility. Advanced Science, 2016, 3: 1600062.

[14] LI XING-XING, WU XIAO-JUN, YANG JIN-LONG. Half-metallicity 
in $\mathrm{MnPSe}_{3}$ exfoliated nanosheet with carrier doping. Journal of the American Chemical Society, 2014, 136(31): 11065-11069.

[15] MUKHERJEE D, AUSTERIA P M, SAMPATH S. Two-dimensional, few-layer phosphochalcogenide, $\mathrm{FePS}_{3}$ : a new catalyst for electrochemical hydrogen evolution over wide $\mathrm{pH}$ range. ACS Energy Letters, 2016, 1(2): 367-372.

[16] DU KE-ZHAO, WANG XING-ZHI, LIU YANG, et al. Weak van der Waals stacking, wide-range band gap, and Raman study on ultrathin layers of metal phosphorus trichalcogenides. ACS Nano, 2015, 10(2): 1738-1743.

[17] ZHANG QIU-HONG, GUO QIANG-BING, CHEN QIAN, et al. Highly efficient 2D NIR-II photothermal agent with fenton catalytic activity for cancer synergistic photothermal-chemodynamic therapy. Advanced Science, 2020, 7(7): 1902576.

[18] FANG XUE-YANG, WU XIAN-LIN, LI ZHEN-DONG, et al. Biomimetic anti-PD-1 peptide-loaded 2D FePSe 3 nanosheets for efficient photothermal and enhanced immune therapy with multimodal MR/PA/thermal imaging. Advanced Science, 2020, 8(2): 2003041

[19] CHENG LIANG, LIU JING-JING, GU XING, et al. PEGylated $\mathrm{WS}_{2}$ nanosheets as a multifunctional theranostic agent for in vivo dual-modal $\mathrm{CT} /$ photoacoustic imaging guided photothermal therapy. Advanced Materials, 2014, 26(12): 1886-1893.

[20] LIN HAN, GAO SHAN-SHAN, DAI CHEN, et al. Two-dimensional biodegradable niobium carbide (MXene) for photothermal tumor eradication in NIR-I and NIR-II bio-windows. Journal of the American Chemical Society, 2017, 139(45): 16235-16247.

[21] COLEMAN J N, LOTYA M, O'NEILL A, et al. Two-dimensional nanosheets produced by liquid exfoliation of layered materials. Science, 2011, 331(6017): 568-571.

[22] ZHAO WEI, LI AI-HUA, ZHANG AI-TANG, et al. Recent advances in functional-polymer-decorated transition-metal nanomaterials for bioimaging and cancer therapy. ChemMedChem, 2018, 13(20): 2134-2149.

[23] ZHANG YONG-CAI, DU ZHEN-NI, LI KUN-WEI, et al. Highperformance visible-light-driven $\mathrm{SnS} / \mathrm{SnO}$ nanocomposite photocatalyst prepared via in situ hydrothermal oxidation of SnS nanoparticles. ACS Applied Materials \& Interfaces, 2011, 3(5): 1528-1537.

[24] CHENG ZHONG-ZHOU, WANG FENG-MEI, SHIFA T A, et al. Efficient photocatalytic hydrogen evolution via band alignment tailoring: controllable transition from type-I to type-II. Small, 2017, 13(41): 1702163.

[25] CHEN PENG, SU YUN, LIU HONG, et al. Interconnected tin disulfide nanosheets grown on graphene for Li-ion storage and photocatalytic applications. ACS Applied Materials \& Interfaces, 2013, 5(22): 12073-12082.

[26] DETTY M R, GIBSON S L, WAGNER S J. Current clinical and preclinical photosensitizers for use in photodynamic therapy. Journal of Medicinal Chemistry, 2004, 47(16): 3897-3915.

[27] SHARMAN W M, ALLEN C M, VAN LIER J E. Photodynamic therapeutics: basic principles and clinical applications. Drug Discovery Today, 1999, 4(11): 507-517.

[28] WANG HUI, YANG XIAN-ZHU, SHAO WEI, et al. Ultrathin black phosphorus nanosheets for efficient single oxygen generation. Journal of the American Chemical Society, 2015, 137(35): 11376-11382.

[29] LIANG CHEN, ZHANG XING-LIN, YANG MENG-SU, et al. Remodeling tumor microenvironment by multifunctional nanoassemblies for enhanced photodynamic cancer therapy. ACS Materials Letters, 2020, 2(10): 1268-1286.

[30] DING XIAN-GUANG, LIOW C H, ZHANG MENG-XIN, et al. Surface plasmon resonance enhanced light absorption and photothermal therapy in the second near-infrared window. Journal of the American Chemical Society, 2014, 136(44): 15684-15693.

[31] BASHKATOV A N, GENINA E A, KOCHUBEY V I, et al. Optical properties of human skin, subcutaneous and mucous tissues in the wavelength range from 400 to $2000 \mathrm{~nm}$. Journal of Physics D: Applied Physics, 2005, 38(15): 2543.

[32] ROBINSON J T, TABAKMAN S M, LIANG YONG-YE, et al. Ultrasmall reduced graphene oxide with high near-infrared absorbance for photothermal therapy. Journal of the American Chemical Society, 2011, 133(17): 6825-6831.

[33] LI K C, CHU H C, LIN Y, et al. PEGylated copper nanowires as a novel photothermal therapy agent. ACS Applied Materials \& Interfaces, 2016, 8(19): 12082-12090.

[34] SUN CAI-XIA, WEN LING, ZENG JIAN-FENG, et al. One-pot solventless preparation of PEGylated black phosphorus nanoparticles for photoacoustic imaging and photothermal therapy of cancer. Biomaterials, 2016, 91: 81-89.

[35] GIORGIO M, MIGLIACCIO E, ORSINI F, et al. Electron transfer between cytochrome $\mathrm{c}$ and $\mathrm{p} 66^{\text {Shc }}$ generates reactive oxygen species that trigger mitochondrial apoptosis. Cell, 2005, 122(2): 221-233.

[36] DANIAL N N, KORSMEYER S J. Cell death: critical control points. Cell, 2004, 116(2): 205-219.

[37] WANG YI, WEI GUO-QING, ZHANG XIAO-BIN, et al. Multistage targeting strategy using magnetic composite nanoparticles for synergism of photothermal therapy and chemotherapy. Small, 2018, 14(12): 1702994. 